\title{
Photonic crystal waveguide-mode orthogonality conditions and computation of intrinsic waveguide losses
}

\author{
Jeremy Witzens, Thomas Baehr-Jones, Michael Hochberg, Marko Lončar, and Axel Scherer \\ Department of Electrical Engineering 136-93, California Institute of Technology, Pasadena, California 91125
}

Received April 14, 2003; revised manuscript received June 17, 2003; accepted June 18, 2003

\begin{abstract}
We simulate the propagation of light in a W1 planar photonic crystal waveguide with the three-dimensional finite-difference time-domain method and apply an inner product against previously calculated mode profiles to the simulated field cross sections. We show that this inner product satisfies mode orthogonality for both photonic crystal waveguides and segmented waveguides and use the obtained data to evaluate waveguide losses. (C) 2003 Optical Society of America
\end{abstract}

OCIS codes: $130.2790,230.3990$.

\section{INTRODUCTION}

Photonic crystals ${ }^{1}$ have attracted considerable attention because of their utility in controlling the flow of light on very small length scales. Planar photonic crystals $(\mathrm{PPCs})^{2}$ represent particularly promising structures for integrated optics since their planar fabrication allows the use of wafer-scale microelectronics fabrication techniques. In particular, line defects formed in a PPC by changing the properties of one or several rows of holes ${ }^{3,4}$ can be used for waveguiding. In such a system the guided wave is confined in the vertical direction by total internal reflection and in the lateral direction by distributed Bragg reflection or confinement based on effective index. Recently, propagation losses as low as $11 \mathrm{~dB} / \mathrm{mm}$ have been reported for silicon-on-insulator-based photonic crystal waveguides. ${ }^{5}$ Losses can be due to material absorption, surface roughness, fabrication tolerance, or intrinsic coupling to free space. The coupling to free space from photonic crystal modes and photonic crystal waveguide modes has been used for light extraction ${ }^{6}$ and experimental mode mapping. ${ }^{7}$ Several experimental ${ }^{8,9}$ and theoretical $^{8-14}$ investigations of the intrinsic losses of photonic crystal waveguides have been published. In this paper we present a novel method for evaluating intrinsic losses with finite-difference time-domain (FDTD) ${ }^{15}$ simulations. Intrinsic photonic crystal waveguide losses have previously been investigated by FDTD simulations, ${ }^{8-14}$ but previous methods using FDTD simulations were based on evaluating the power transmission of a device ${ }^{16}$ or the intensity decay along the waveguide. ${ }^{8,14}$ In both cases, distinguishing insertion losses due to mode mismatch from propagation losses is difficult. In the first case, several simulations need to be done to find the asymptotic value of losses as a function of device length (and to ensure that there is no tunneling), and in the second case the simulation needs to be propagated for a longenough distance to ensure that the field has converged to a waveguide mode. Both methods require simulations on the order of the length scale required for the field to con- verge to a mode. In Ref. 14, mode profiles are calculated but are not used for the propagation-loss calculations. We propose to propagate the field for a short distance (a few lattice constants) and to use previously calculated mode profiles to filter out the fraction of the field that belongs to the waveguide mode. We will show that a conserved functional commonly used as an inner product for continuous waveguides can also be used for photonic crystal waveguides and segmented waveguides.

We analyze a W1 waveguide formed by leaving out one row of holes in a triangular lattice planar photonic crystal of lattice parameter $a$, radius $r=0.3 a$, and slab thickness $t=0.577 a .^{8}$ The slab has an index of 3.43, and the holes and the cladding have an index of 1 . The photonic crystal is in the $x y$ plane, and the direction of propagation is $y$. We evaluate the losses for the 1 mode, which has even symmetry relative to the $x y$ plane $\left(\sigma_{x y}=+1\right)$ and odd symmetry relative to the $y z$ plane $\left(\sigma_{y z}=-1\right)$. All simulations are performed by three-dimensional (3D) FDTD with a discretization of 26 points per lattice parameter $a$. In order to get stable simulations with Bloch boundary conditions (BBCs), the time step $d t$ was reduced to $d x / 4$ (in units of $c_{0}=1$ ), where $d x$ is the spatial discretization and $c_{0}$ is the speed of light in vacuum. All results are given in normalized frequency $a / \lambda$ and normalized wave vector $a k$, where $\lambda$ is the wavelength and $k$ the wave vector of light in vacuum.

To obtain the modal profiles of the photonic crystal waveguide, we simulate one unit cell of the waveguide, with $\mathrm{BBC}$ in the direction of propagation ( $y$ axis). ${ }^{17}$ The $\mathrm{BBC}$ forces the field on the $y=a$ boundary to be $\exp (i \phi)$ times the field on the $y=0$ boundary, where $\phi$ is a fixed phase. Through discrete Fourier transform applied on the time series collected by a field probe, the spectral resonances are found and filtered out. In this way, modes and leaky modes of the photonic crystal waveguide are obtained. These simulations will be referenced hereafter as "mode solving" in this article. We save the modal field profile along an $x z$ plane. For all that follows, the 
specific position of the $x z$ plane is not important as long as it is consistent. In order to obtain the forwardpropagating mode, the $\mathrm{BBC}$ is set to a phase $-k a$, since the dispersion diagram has negative slope (the group velocity points in the direction opposite to $k$ ). Alternatively, the field could also be solved with a BBC of $k a$, followed by a field transformation that inverts propagation direction with respect to $y^{18}$ :

$$
\begin{aligned}
& \left(E_{x}, E_{y}, E_{z}\right) \rightarrow\left(E_{x},-E_{y}, E_{z}\right)^{*}, \\
& \left(B_{x}, B_{y}, B_{z}\right) \rightarrow\left(-B_{x}, B_{y},-B_{z}\right)^{*} .
\end{aligned}
$$

We then simulate field propagation along ten photonic crystal periods. Figure 1 shows the simulation domain. We use dipole sources to launch a pulse into the photonic crystal waveguide and save the field profile every second lattice period at the same position relative to the unit cell as in the mode solve. We perform a discrete Fourier transform on the field cross section and extract the frequencies corresponding to the photonic crystal waveguide modes of interest.

We then use an inner product to filter out the portion of the field that is in the e 1 mode and evaluate the waveguide losses. In Section 2 the inner product is introduced, and in Section 3 we show the simulation results.

\section{INNER PRODUCT}

For waveguides with continuous symmetry, orthogonality conditions between bound modes and between bound modes and radiative modes are well established. ${ }^{18}$ In particular, for a nonabsorbing waveguide with translation symmetry in the $y$ direction and two modes (bound or radiative) $\psi_{j}=(\mathbf{E}, \mathbf{H})$ and $\left(\bar{\psi}_{k}=\overline{\mathbf{E}}, \overline{\mathbf{H}}\right)$ with the same implicit time dependence $\exp (-i \omega t)$,

$$
\begin{array}{ll}
\mathbf{E}=\mathbf{e}_{j}(x, z) \exp \left(i \beta_{j} y\right), & \mathbf{H}=\mathbf{h}_{j}(x, z) \exp \left(i \beta_{j} y\right), \\
\overline{\mathbf{E}}=\mathbf{e}_{k}(x, z) \exp \left(i \beta_{k} y\right), & \overline{\mathbf{H}}=\mathbf{h}_{k}(x, z) \exp \left(i \beta_{k} y\right),
\end{array}
$$

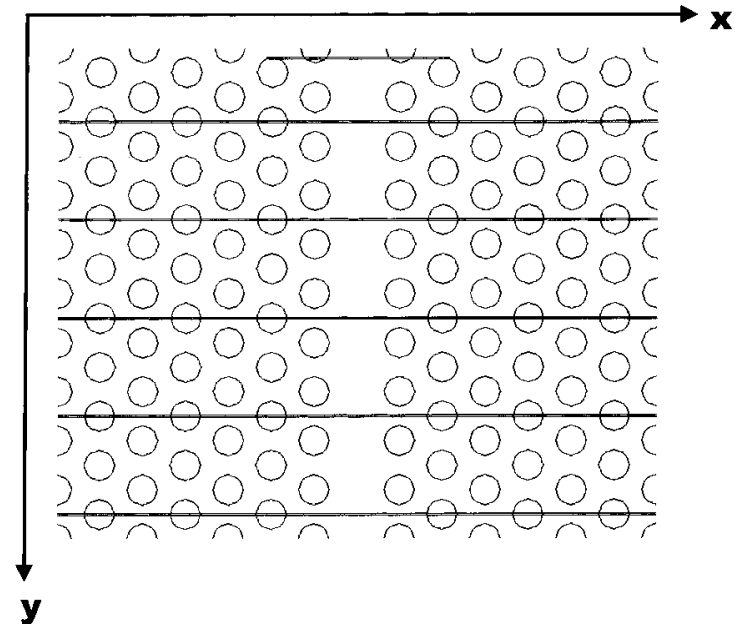

Fig. 1. Cross section of the simulation domain. A dipole source launches a field of symmetry $\sigma_{x y}=-1, \sigma_{y z}=+1$. Every second lattice period a field probe saves the field. where $\omega$ is the angular frequency and $\beta_{j / k}$ are the propagation constants, the following holds:

$$
\left(\beta_{j}-\beta_{k}\right) \int_{A}\left\{\mathbf{e}_{j} \times \mathbf{h}_{k}^{*}+\mathbf{e}_{k}^{*} \times \mathbf{h}_{j}\right\} \cdot \hat{\mathbf{y}} \mathrm{d} A=0,
$$

where $\hat{\mathbf{y}}$ is the normal vector in the $y$ direction and $A$ is an $x z$ plane. Photonic crystal waveguide modes can be written as

$$
\begin{aligned}
& \mathbf{E}=\mathbf{e}_{j}(x, y, z) \exp \left(i \beta_{j} y\right), \\
& \mathbf{H}=\mathbf{h}_{j}(x, y, z) \exp \left(i \beta_{j} y\right), \\
& \overline{\mathbf{E}}=\mathbf{e}_{k}(x, y, z) \exp \left(i \beta_{k} y\right), \\
& \overline{\mathbf{H}}=\mathbf{h}_{k}(x, y, z) \exp \left(i \beta_{k} y\right),
\end{aligned}
$$

where $\beta_{j}$ and $\beta_{k}$ are in the first Brillouin zone and $\mathbf{e}_{j / k}$ and $\mathbf{h}_{j / k}$ are periodic in the $y$ direction with the lattice constant $a$ as a period. We use the same functional as an inner product for photonic crystal waveguides:

$$
\langle\psi \mid \bar{\psi}\rangle_{y_{0}}=\frac{1}{4} \int_{A\left\{y=y_{0}\right\}}\left\{\mathbf{e}_{j} \times \mathbf{h}_{k}^{*}+\mathbf{e}_{k}^{*} \times \mathbf{h}_{j}\right\} \cdot \hat{\mathbf{y}} \mathrm{d} A .
$$

This inner product is not a scalar product, because backward-propagating modes have a negative square product and standing waves are null vectors. However, for a steady-state field $|\psi\rangle,\langle\psi \mid \psi\rangle_{y_{0}}$ is the time-averaged power flux propagating in the $y$ direction and is thus independent of the reference plane $A\left\{y=y_{0}\right\}$ (we assumed nonabsorbing materials and $A$ as an infinite plane). We apply Fourier filtering before calculating the inner products. In particular, the transfer matrix $T_{y_{0} \rightarrow y_{0}+a}=T_{a}$ that maps the field from one cross section $A_{1}\left\{y=y_{0}\right\}$ to a cross section $A_{2}\left\{y=y_{0}+a\right\}$, which is one lattice parameter farther along the $y$ direction, conserves $\langle\psi \mid \psi\rangle$. From the conservation of the square product we conclude conservation of the inner product:

$$
\begin{aligned}
\left\langle\psi_{1} \mid \psi_{2}\right\rangle_{y_{0}} & =\left\langle\psi_{1} \mid \psi_{2}\right\rangle_{y_{0}+a}, \\
\left\langle\psi_{1} \mid \psi_{2}\right\rangle & =\left\langle\psi_{1}\left|T_{a}^{+} T_{a}\right| \psi_{2}\right\rangle .
\end{aligned}
$$

The EM field can be decomposed into eigenmodes of the operator $T_{a}$ (Bloch modes) because of the periodicity of the photonic crystal waveguide (Bloch theorem). For two modes $\left|\psi_{1}\right\rangle$ and $\left|\psi_{2}\right\rangle$ satisfying $T_{a}\left|\psi_{1}\right\rangle=\exp \left(i k_{1} a\right)\left|\psi_{1}\right\rangle$ and $T_{a}\left|\psi_{2}\right\rangle=\exp \left(i k_{2} a\right)\left|\psi_{2}\right\rangle$, we have

$$
\left\langle\psi_{1}\left|T_{a}^{+} T_{a}\right| \psi_{2}\right\rangle=\exp \left[i\left(k_{2}-k_{1}\right) a\right]\left\langle\psi_{1} \mid \psi_{2}\right\rangle .
$$

From Eqs. (9) and (10) we conclude that $\left\langle\psi_{1} \mid \psi_{2}\right\rangle=0$ if $k 1 \neq k 2$. When two bands cross, we can conclude from the continuity of the scalar product that $\left\langle\psi_{1} \mid \psi_{2}\right\rangle=0$ also holds at the crossover point. However, the method used for mode solving in this paper does not discriminate between degenerate modes (same wave number and same frequency). The solution obtained is then a superposition of the two degenerate modes. One way to obtain the mode profiles would be to run the mode solve twice with different initial fields and then find the superpositions of those two solutions that correspond to the modes. Let us call the two crossing bands band 1 and band 2 . Because the mode profiles are a continuous function of frequency (a result obtained from solid-state band theory) the scalar 
product between the mode of band 1 at the crossover point and a mode of band 2 at a different frequency (where there is no degeneracy and the mode can be readily obtained) tends to zero when that frequency offset tends to zero. We can thus obtain a mode of band 2 at a frequency slightly offset from the crossover point, but offset enough that the modes of the two bands can be distinguished, and then use as a discrimination criterion that the mode of band 1 at the crossover point should be orthogonal to the mode of band 2. This method is more accurate if the mode of band 2 is very close to the crossover point. How close it can be taken to that point depends primarily on the spectral resolution given by the number of time steps used in the mode solve. Another solution could consist in using directly the field profile obtained from the mode solve (which is a superposition of the two degenerate modes) and then fitting the data obtained with this field profile with a sum of two exponentials instead of fitting it with a single exponential. This latter method would be particularly appropriate if the losses of the two modes were very distinct, in which case the separation into two exponentials should then be straightforward.

\section{RESULTS}

The simulation domain is $7 \sqrt{(3)} a \times 3.84 a$ in the $x z$ plane and $10 a$ in the $y$ direction. The properties of the inner product (orthogonality and flux conservation) have been derived for an integral over an infinite plane. When inner products are taken with bound modes, the integration domain can be reduced to a finite cross section because of the exponential decay of the bound modes in the evanescent field region. However, this does not hold for radiative modes. In particular, $\langle\psi \mid \psi\rangle$ is not conserved along the $y$ direction, because radiative modes are absorbed by the perfectly-matched-layer absorbing boundary condition.

The same simulation could have been performed with a $\mathrm{BBC}$ with a phase of 0 applied to the $x y$ and $y z$ boundaries. This would create a supercell in which bound modes of the several layers of PPC waveguide are coupled to each other. If the simulation domain is big enough in the $x$ and $z$ dimensions, however, these couplings are negligible. In such a case we would expect $\langle\psi \mid \psi\rangle$ to be conserved.

We analyze losses for the e1 mode between the light line and the point where the dispersion is folded back a second time into the Brillouin zone (at the $\Gamma$ point). At that point a mini stop band opens. ${ }^{8}$ Figure 2 shows $\left|B_{z}\right|$ for the e1 mode at $a / \lambda=0.3383(k a=0.07 \pi)$. The field amplitude has two maxima, but they have the same phase (i.e., $B_{z}$ is even and $\sigma_{y z}=-1$ ). Figure 3 shows $20 \log _{10}\left(\left|\left\langle\psi_{k a=0.07 \pi} \mid \psi_{l}\right\rangle\right|\right)$, where $\left|\psi_{k a=0.07 \pi}\right\rangle$ is the photonic crystal waveguide mode and $\left|\psi_{l}\right\rangle$ is the field profile at the $l$ th probe and at $a / \lambda=0.3383$. The field intensity shows the exponential decrease that is to be expected. On the same plot $10 \log _{10}\left(\left|\left\langle\psi_{l} \mid \psi_{l}\right\rangle\right|\right)$ is also shown. It can be seen that using the latter would result in an overevaluation of the losses because the field decay is partially due to insertion losses and the field has not yet converged to the photonic crystal waveguide mode. If the lattice parameter $a$ is chosen to be $0.52 \mu$, the intrinsic losses are $110 \mathrm{~dB} / \mathrm{mm}$ at that frequency.

As an additional test we plotted the phase of $\left\langle\psi_{k a=0.07 \pi} \mid \psi_{l}\right\rangle$ (Fig. 4). It can be seen that it has the expected linear behavior and that it corresponds to a wave vector of $0.0698 \pi$ (which is very close to $0.07 \pi$ fixed in the mode solve).

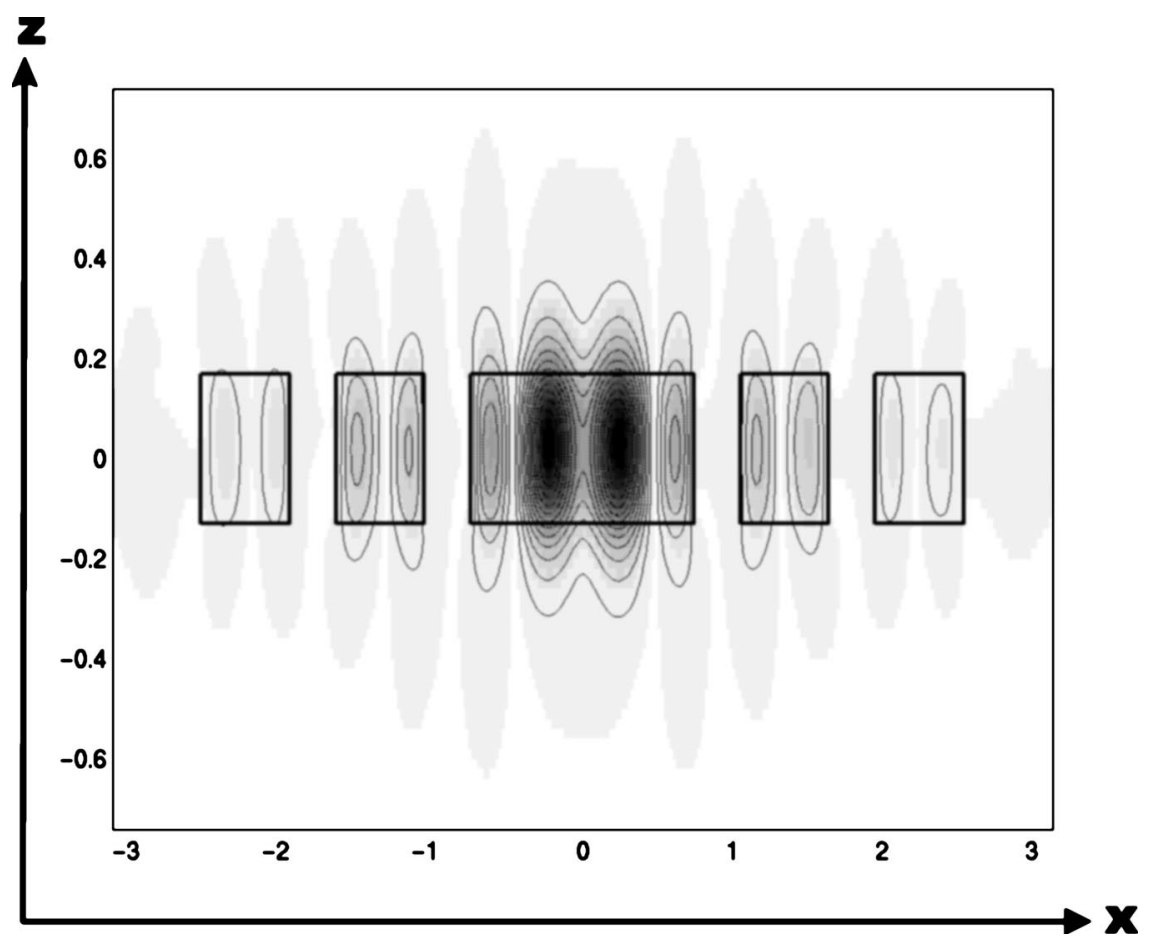

Fig. 2. Mode profile for $k a=0.07 \pi$ (lower band of e1). The colormap shows $\left|B_{z}\right|$. The thick black line shows the high-index region $(n=3.43)$. The two maxima have the same phase (i.e., $B_{z}$ is even). Units are in micrometers for a pitch of $a=0.52 \mu$. 


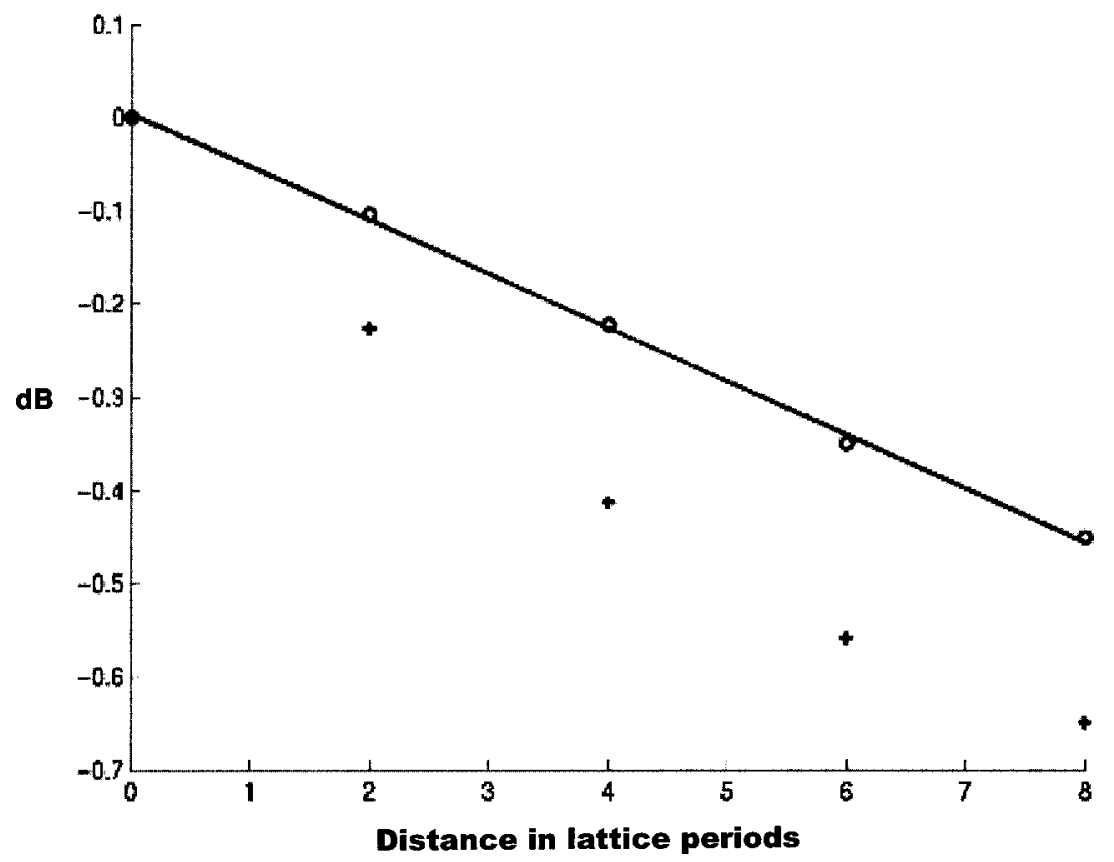

Fig. 3. $20 \log _{10}\left(\left|\left\langle\psi_{l} \mid \psi_{k a=0.07 \pi}\right\rangle\right|\right)$ is plotted with circles, where $\left|\psi_{l}\right\rangle$ is the field profile at the $l$ th probe and $\left|\psi_{k a=0.07 \pi}\right\rangle$ is the mode profile at $k a=0.07 \pi . \quad 10 \log _{10}\left(\left|\left\langle\psi_{l} \mid \psi_{l}\right\rangle\right|\right)$ is plotted with crosses. Probes are spaced by two lattice constants.

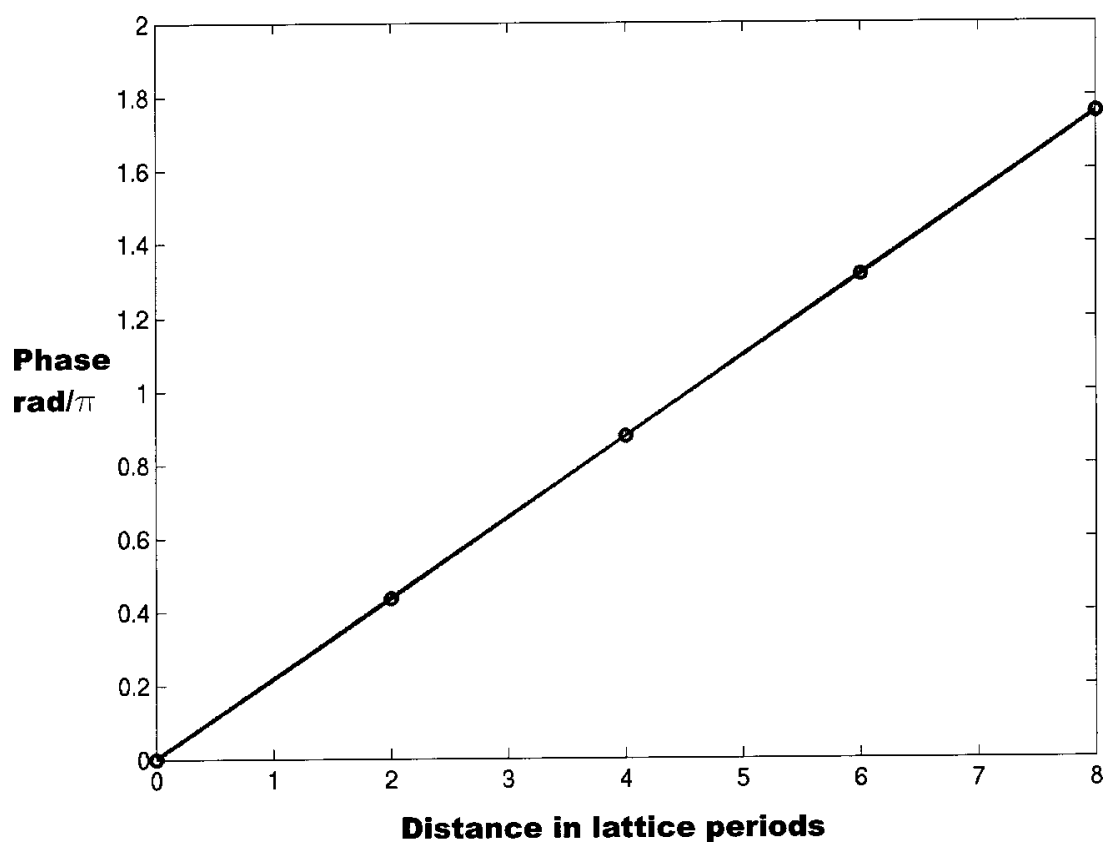

Fig. 4. Plot of the phase of $\left\langle\psi_{l} \mid \psi_{k a=0.07 \pi}\right\rangle$. The expected linear behavior is seen.

Figure 5 shows the computed waveguide losses and the dispersion diagram for the lower band of the e1 mode. Losses first increase away from the light line but then decrease again in the vicinity of the mini stop band.

This numerical method used should be very accurate in the region of high group velocity (from $a / \lambda=0.28$ to $a / \lambda$ $=0.33$ ). However, this method cannot be used to calculate the quality factor of zero group-velocity modes, because these modes are null vectors for the inner product used. For modes of increasingly small group velocity, numerical inaccuracies in the mode profile that have a finite flux become dominant and lead to inaccurate results. To show this, we compared for each data point the wave vector extracted from the inner products with the wave vector initially fixed in the mode-solve procedure (Fig. 6). It is seen that those values correspond for modes away from the mini stop band. However, the first eight data points from $k a=0$ to $k a=0.04 \pi$ show a discrepancy. Although the field profiles of the computed modes look good (comparable with those of Fig. 2), the numerical error that is associated with forward-propagating power dominates the inner products. Consequently, we did not plot those eight data points on Fig. 5.

The decrease of radiative losses for the lower band of e1 


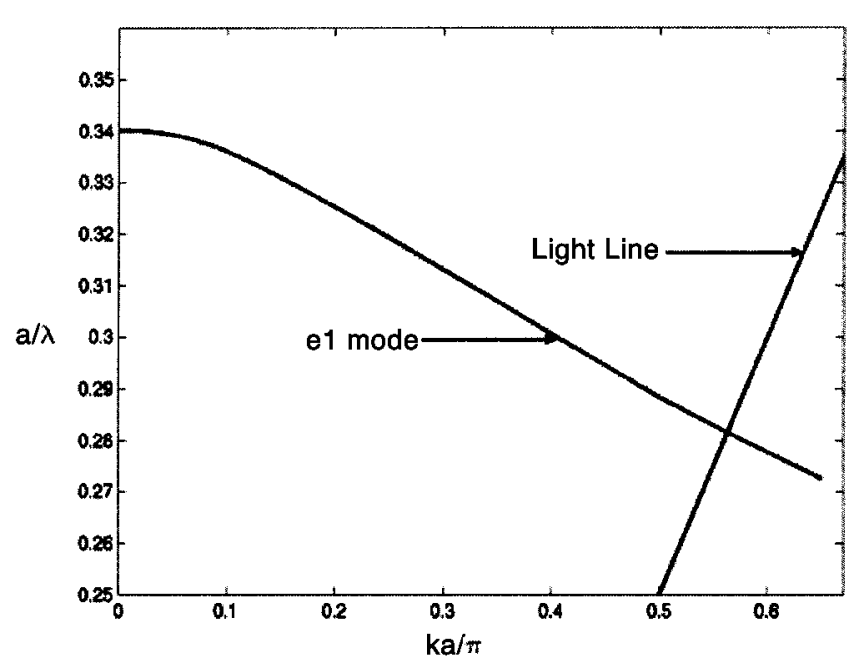

(a)

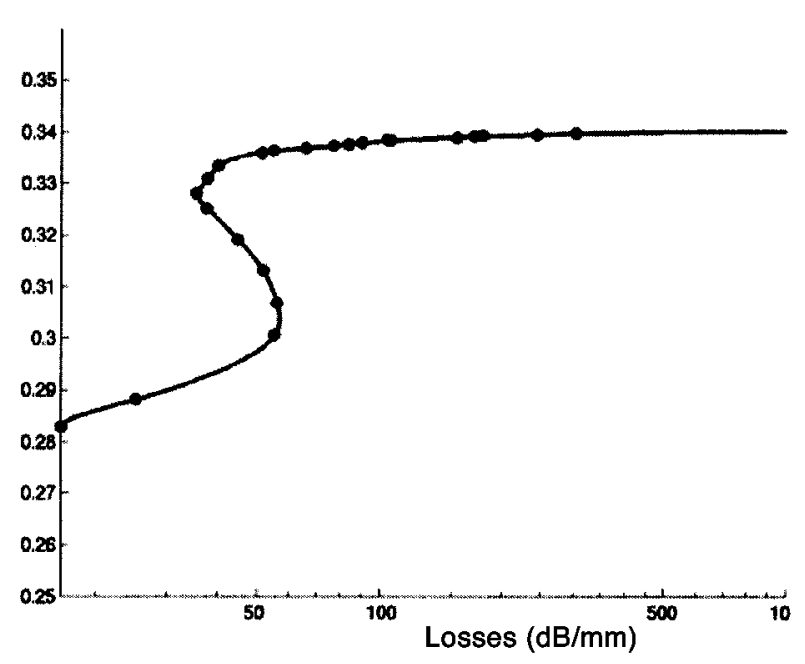

(b)

Fig. 5. (a) Dispersion diagram of the lower band of e1, (b) losses of the lower band of e1. The straight line in (a) represents the light line. Losses (in $\mathrm{dB} / \mathrm{mm}$ ) correspond to a lattice parameter of $a=0.52 \mu$.

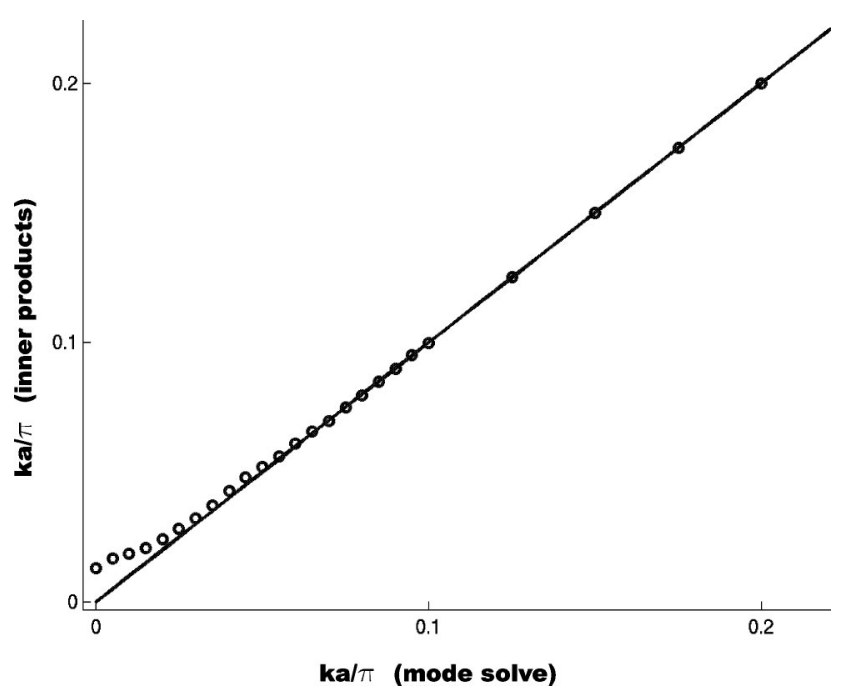

Fig. 6. $\quad k a$ as set in the mode solve versus $k a$ extracted from the phases of $\left\langle\psi_{l} \mid \psi_{k a}\right\rangle$. Away from the mini stop band, in the highgroup-velocity regime, there is a good correspondence. However, near the mini stop band the behavior of phase $\left\langle\psi_{l} \mid \psi_{k a}\right\rangle$ differs from what would be expected from the dispersion diagram.

when the mini stop band is approached has been experimentally observed ${ }^{8}$ and theoretically investigated by other methods. ${ }^{13}$ Reference 13 is based on a twodimensional effective-index analysis. In that model, an upturn of losses in the immediate vicinity of the mini stop band is also observed but in a frequency range too narrow to be experimentally resolved, whereas in our analysis the upturn occurs between $a / \lambda=0.33$ and $a / \lambda=0.34$. It is not completely clear whether this is an artifact of the low group velocity and the limitations of the inner product or is due to $3 \mathrm{D}$ behavior.

\section{CONCLUSION}

We have shown that an inner product usually applied to waveguides of continuous symmetry also verifies the or- thogonality condition between photonic crystal waveguide modes. The inner product was used to analyze data from an FDTD simulation and to evaluate losses of a W1 waveguide. General trends exposed in the previous literature are verified. However, the accuracy of the method is limited for modes of very low group velocity.

Corresponding author Jeremy Witzens can be reached by e-mail at witzens@caltech.edu.

\section{REFERENCES}

1. E. Yablonovitch, "Inhibited spontaneous emission in solidstate physics and electronics," Phys. Rev. Lett. 58, 20592062 (1987).

2. T. F. Krauss, R. M. De La Rue, and S. Brand, "Twodimensional photonic-bandgap structures operating at near infrared wavelengths," Nature (London) 383, 699-702 (1996).

3. A. Mekis, J. C. Chen, I. Kurand, S. Fan, P. R. Villeneuve, and J. D. Joannopoulos, "High transmission through sharp bends in photonic crystal waveguides," Phys. Rev. Lett. 77, 3787-3790 (1996).

4. T. Baba, N. Fukaya, and J. Yonekura, "Observation of light propagation in photonic crystal optical waveguides with bends," Electron. Lett. 35, 654-655 (1999).

5. T. Baba, A. Motegi, T. Iwai, N. Fukaya, Y. Watanabe, and A. Sakai, "Light propagation characteristics of straight singleline-defect waveguides in photonic crystal slabs fabricated into a silicon-on-insulator substrate," IEEE J. Quantum Electron. 38, 743-752 (2002).

6. M. Boroditsky, R. Vrijen, T. F. Krauss, R. Coccioli, R. Bhat, and E. Yablonovitch, "Spontaneous emission extraction and Purcell enhancement from thin-film 2-D photonic crystals," J. Lightwave Technol. 17, 2096-2112 (1999).

7. V. N. Astratov, I. S. Culshaw, R. M. Stevenson, D. M. Whittaker, M. S. Skolnick, T. F. Krauss, and R. M. De la Rue, "Resonant coupling of near-infrared radiation to photonic band structure waveguides," J. Lightwave Technol. 17, 2050-2057 (1999).

8. M. Lončar, D. Nedeljković, T. P. Pearsall, J. Vučković, A. Scherer, S. Kuchinsky, and D. C. Allan, "Experimental and theoretical confirmation of Bloch-mode light propagation in planar photonic crystal waveguides," Appl. Phys. Lett. 80, 1689-1691 (2002). 
9. H. Benisty, P. Lalanne, S. Olivier, M. Rattier, C. J. M. Smith, T. F. Krauss, C. Jouanin, and D. Cassagne, "Finitedepth and intrinsic losses in vertically etched twodimensional photonic crystals," IEEE J. Quantum Electron. 35, 205-215 (2002).

10. P. Lalanne, "Electromagnetic analysis of photonic crystal waveguides operating above the light cone," IEEE J. Quantum Electron. 38, 800-804 (2002).

11. P. Lalanne and H. Benisty, "Out-of-plane losses of twodimensional photonic crystals waveguides: electromagnetic analysis,” J. Appl. Phys. 89, 1512-1514 (2001)

12. G. R. Hadley, "Out-of-plane losses of line defect photonic crystal waveguides," IEEE Photonics Technol. Lett. 14, 642-644 (2002).

13. L. C. Andreani and M. Agio, "Intrinsic diffraction losses in photonic crystal waveguides with line defects," Appl. Phys. Lett. 82, 2011-2013 (2003).
14. Y. Désières, T. Benyattou, R. Orobtchouk, A. Morand, P. Benech, C. Grillet, C. Seassal, X. Letartre, P. Rojo-Romeo, and P. Viktorovitch, "Propagation losses of the fundamental mode in a single line-defect photonic crystal waveguide on an InP membrane," J. Appl. Phys. 92, 2227-2234 (2002).

15. A. Taflove, Computational Electrodynamics: the FiniteDifference Time-Domain Method (Artech House, Norwood, Mass., 1995).

16. M. Qiu, B. Jaskorzynska, M. Swillo, and H. Benisty, "Timedomain 2D modeling of slab-waveguide-based photoniccrystal devices in the presence of radiation losses," Microwave Opt. Technol. Lett. 34, 387-393 (2002).

17. M. Lončar, T. Doll, J. Vučković, and A. Scherer, "Design and fabrication of silicon photonic crystal optical waveguides," J. Lightwave Technol. 18, 1402-1411 (2000).

18. A. W. Snyder and J. D. Love, Optical Waveguide Theory (Chapman \& Hall, London (1983). 